Zuerst ersch. in: Kompetenz, Kognition und neue Konzepte der beruflichen Bildung / Philipp Gonon ... (Hrsg.). Wiesbaden: VS Verlag für Sozialwissenschaften, 2005, S. 237-251

\section{Lernmotivation in lehrer- und schülerzentrierten Unterrichtssequenzen - Analyse des Unterrichtserlebens mit Hilfe von Selbstberichts- und Videodaten}

\section{Jürgen Seifried}

\section{Problemstellung}

$\mathrm{Zu}$ einer wichtigen Grundbedingung erfolgreicher Berufsausbildung zählt motiviertes Lernen. Da dem Status einer abgeschlossenen Berufsausbildung heute eher ein temporărer Charakter zukommt, ist motiviertes Lernen darüber hinaus als Gelingensbedingung für Lebenslanges Lernen zu betrachten (Sembill 2000). Im Hinblick auf die vermutete Bedeutung von motiviertem Lernen für den (Aus-)Bildungserfolg ist es somit unmittelbar von Interesse, wie sich Motivationslagen von Lernenden darstellen - und zwar sowohl für jedes Individuum als auch für Gruppen oder gesamte Klassenverbände. Zudem ist für das pădagogische Handeln von Bedeutung, wie Kontextbedingungen von den Lernern wahrgenommen werden und wie diese sich auf Lernmotivation auswirken. Schließlich gilt es, durch den Einbezug von Lernervariablen zu einem besseren Verständnis von Motivationsprozessen zu gelangen.

Vor dem Hintergrund obiger Ausführungen erstaunt es kaum, dass das Thema Motivation/Lernmotivation z. B. bei Fachtagungen einen breiten Raum einnimmt. Vergleichsweise geringe Bedeutung wurde bisher jedoch der Untersuchung motivationaler Prozesse sowie deren Abhängigkeit von situativen Bedingungen beigemessen. Dies wird $u$. a. begrundet mit methodischen Schwierigkeiten bei der Analyse solcher Prozesse in natiurlichen Lernumgebungen sowie mit Problemen, die mit der Gewinnung entsprechender empirischer Daten - ausreichend große Stichprobe, Untersuchung von Motivationsprozesse über längere Aus- oder Weiterbildungszeiträume - verbunden sind (Wild \& Krapp 1996, S. 196 f.). Ein wesentliches Manko derzeitiger Forschung zur Lernmotivation besteht darin, „dass die - in der Regel längsschnittlich gewonnenen - Zusammenhänge zwischen Fragebögen zur Erfassung motivationaler Orientierungen und kumulativen Leistungsmaßen nur wenig zum Verständnis der vermittelnden motivationalen und kognitiven Prozesse aussagen" (Wild 2000, S. 39). Untersuchungen, die den genannten 
Problemlagen Rechnung tragen und explizit auf die Analyse von Motivationsprozessen abzielen, sind nur selten zu finden.'

Möchte man einen vertieften Einblick in motivationale Prozesse erlangen, so. stellt die - zugegebenermaßen recht aufwendige - empirische Koppelung verschiedener Analyseebenen eine Erfolg versprechende Möglichkeit dar. Hierzu wird ein mehrstufiges Erhebungs- und Auswertungsverfahren notwendig: ${ }^{2}$

(1) Einen ersten Zugang zur Analyse von Lernprozessen bietet das Verhalten der am Unterrichtsgeschehen Beteiligten. Hierzu dient die Auswertung von Videodaten.

(2) Neben aus der Dokumentation des Unterrichts gewonnenen Informationen stellen Selbstberichte zum subjektiven Erleben der Lehr-LernSituationen aus Sicht der beteiligten Schülerinnen und Schüler eine bedeutsame Erkenntnisquelle dar. Hierzu sind Informationen zu bewuss erlebten motivationalen Zustănden zu erheben. ${ }^{3}$ Zur Gewinnung empirischer Daten $\mathrm{zu}$,frequency, intensity, and patterning of psychological states, i. e., emotional, cognitive, and conative dimensions of experience" (Csikszentmihalyi \& Larson 1987, S, 526) wird seit Mitte der 1970er Jahre die Experience-Sampling Method (ESM) eingesetzt (Csikszentmihalyi \& Larson 1987). Dabei tragen Personen, während sie ihren alltäglichen Tätigkeiten nachgehen, ein Signalgerät bei sich, das nach einem Zeitstichprobenplan aktiviert wird. Der Proband wird dann zur Beschreibung der aktuellen Handlungen, des emotionalen und/oder kognitiven Erlebenszustandes sowie zur Charakterisierung des Kontextes aufgefordert. Als besonderer Vorteil des Verfahrens wird die ökologische Validität der Selbstberichte betrachtet: Das unmittelbare erichten von Emotionen und psychischen Zuständen macht die i. d. R. weniger zuverlässige retrospektive Schilderung überflüssig.

1 Ausnahmen bilden beispielsweise die Studien von Wild \& Krapp 1996; Wild 2000; Seidel 2003 sowie die Arbeiten von Sembill et al. (Sembill 1996, 2000; Sembill, Wolf, Wuttke Wolf, Santjer \& Schumacher 1998; Wuttke 1999; Sembill, Schumacher, Wolf, Wuttke \& Santjer-Schnabel 2001; Santjer-Schnabel 2002, Schumacher 2002; Wolf 2003; Seifried 2004a, 2004b)

2 Zudem können Lernprozesse mit Hilfe (neuro-)physiologischer Daten erfasst werden. Die Aufzeichnung von Verănderungen der Hautleitfăhigkeit oder Herzfrequenz ermoglicht bejspielsweise Rückschlüsse auf das Erleben von Lehr-Lern-Situationen. Im Rahmen der von der Forschergruppe um Sembill durchgefuhrten Prozessanalysen Selbstorganisierten Lernen hat Santjer-Schnabel (2002) umfangreiche Auswertungen vorgelegt, die Hinweise auf den Zusammenhang zwischen physiologischen Daten und Selbstberichten zu emotionalen, kognitiven und motivationalen Zustănden geben.

3 Dabei gilt es zu bedenken, dass nur ein geringer Teil des Verhaltens von bewusstem Erleben begleitet wird (Roth 2001; Damasio 1999).
(3) Die empirische Koppelung der genannten Ebenen ermöglicht die Identifizierung von Zusammenhangsmustern zwischen der sichtbaren Handlungsebene (,Außensicht") und dem nicht unmittelbar beobachtbaren subjektiven Erleben des Unterrichts („Innensicht"). Mit Außensicht sind dabei die beobachtbaren Muster der Unterrichtsorganisation gemeint (z. B. unterrichtliche Arbeitsformen und Lerninhalte), wohingegen der Begriff Innensicht die Einschătzungen und Beurteilungen der Schliler bezüglich des Unterrichts beschreibt.

Bei den in der Vergangenheit durchgeführten Prozessanalysen Selbstorganisierten Lernens ${ }^{4}$ konnte durch längsschnittlich angelegte Vergleiche von traditionell und selbstorganisiert lernenden Untersuchungsgruppen mehrfach nachgewiesen werden, dass sich eine selbstorganisationsoffene Lernumgebung günstig auf Lernmotivation und das kognitive Engagement von Lernenden auswirkt. Es stellt sich nun die Frage, inwieweit sich für verschiedene ,activity structures“ (Berliner 1983) innerhalb einer selbstorganisationsoffenen Lernumgebung Unterschiede ergeben. Dabei ist zu vermuten, dass Schüler in Einzel- oder Gruppenarbeitsphasen eine höhere Aktivierung aufweisen als im Frontalunterricht. Dies wird in einschlägigen didaktischen Kompendien (z. B. Meyer 1994) regelmäßig postuliert; nach wie vor mangelt es jedoch an empirischen Nachweisen (Wild 2000, S. 41).

Weiterhin ist von Interesse, ob das Unterrichtserleben in den verschiedenen Unterrichtsphasen von der individuellen Konstitution der Lernenden beeinflusst wird. Unter Rückgriff auf Ergebnisse aus der ATI-Forschung (siehe hierzu Helmke \& Weinert 1997, S. 140 f.) ist beispielsweise zu vermuten, dass Schüler mit Nachteilen hinsichtlich der emotional-motivationalen Konstitution eher von den klaren Strukturen einer lehrer-geleiteten Instruktion und leistungsstärkere oder weniger ängstliche Schüler von offenem Unterricht profitieren. In der Lehr-Lern-Forschung werden diesbezüglich jedoch widersprüchliche Befunde berichtet. Wăhrend beispielsweise Nickolaus \& Bickmann (2002) für handlungsorientiert unterrichtete schwache Klassen Nachteile konstatieren, ergeben sich im Rahmen der Münchner Haupt- und Grundschulstudie keine derartigen Effekte (Helmke \& Weinert 1997, S. 142). Die bisher durchgefuhrten Prozessanalysen Selbstorganisierten Lernens von Sembill et al. (s. o.) geben ebenfalls Anlass zu der Vermutung, dass offener Unterricht nicht zwangsläufig zu einer Benachteiligung schwächerer Schüler führt.

4 Aufbauend auf reformpaddagogischen Ansätzen weist Selbstorganisiertes Lernen nach Sembill eine hohe Affinitat zum Projektunterricht im ursprunglichen Sinne auf und zeichnet sich durch eine umfassende Übertragung von Lernverantwortung auf Lernende aus. Kurz gefasst eht es darum, in projektorientierter Kleingruppenerbeit in eigener Veroutwortung uber men$\mathrm{m}$, in projektorientierter Kleingruppenarbeit in eigener Verantwortung uber methrere Unterrichtsstunden hinweg komplexe, praxisnahe Problemstellungen zu bearbeiten. 


\section{Konzeption der empirischen Untersuchung}

Der vorliegende Beitrag widmet sich folgenden Fragestellungen:

(1) Es geht zunächst um das motivationale Erleben des Unterrichts in $\mathrm{Ab}$ hängigkeit von Unterrichtsgegebenheiten. Im Fokus des Interesses steht hier insbesondere die unterrichtliche Arbeitsform. Zur Beantwortung dieser Fragestellungen greifen wir auf Selbstberichtsdaten zum subjektiven Erleben des Rechnungswesenunterrichts zuruck und stellen diese für verschiedene Formen der Unterrichtsorganisation gegenüber.

(2) In einem zweiten Analyseschritt wird untersucht, ob das Unterrichtserleben in Abhängigkeit der individuellen Konstitution von Lernenden variiert.

Datenbasis

Die im Folgenden darzustellenden empirischen Befunde gehen auf die $\mathrm{Ge}$ genüberstellung zweier in einer selbstorganisationsoffenen Lernumgebung (Selbstorganisiertes Lernen: SoLe) unterrichten Klassen zurück, die sich sich hinsichtlich der fachdidaktisch-curricularen Ausrichtung unterscheiden. Während Klasse $1(n=22)$ herkömmlichen Rechnungswesenunterricht (Bilanzmethode) erhielt (kurz: SoLe + Bil.), wurde eine zweite Klasse $(n=22)$ nach den Prinzipien des wirtschaftsinstrumentellen Rechnungswesens (Preiß 1999) unterrichtet (kurz: SoLe + WiR). ${ }^{5}$

Erfassung des subjektiven Erlebens des Unterrichts (,Innensicht“)

Um das motivationale Erleben in den beiden Klassen adäquat zu erfassen, kamen portable Datenerfassungsgeräte zum Einsatz. Damit vermag man das Prozesserleben in beliebig kurzer Taktung zu messen, während die Probanden einer alläglichen Beschäftigung nachgehen, nämlich dem Lernen während des Berufsschulbesuchs. Die Versuchspersonen sollten jeweils ihren internen Zustand sowie den Zustand der Umwelt bezogen auf das eigene Subjekt einschătzen, indem sie auf einer stufenlosen Skala von 0 bis $100 \mathrm{u}$. a den Grad der Zustimmung zu folgenden Items angeben: „Ich kann mitgestal-

5 Durch den Einbezug einer sowohl hinsichtlich der Lehr-Lern-Methodik (fragendDrich lanzons sagen zur prinzipiellen Eignung des Selbstorganisierten Lernens fur den Rechnungswesenunterricht möglich sind. Als ein zentrales Ergebnis der Auswertungen zeigt sich, das Schuler, die in einer selbstorganisationsoffenen Lernumgebung unterrichtet werden, insbesondere hinsichtlich der Problemlösefáhigkeit überlegene Resultate erzielen (Seifried, Brouer \& Sembill 2002; Seifried 2004a). Damit kơnnen die in den bereits durchgefuhrten Untersuchungen zum Selbstorganisierten Lernen gewonnenen Befunde zur Problemlösekompetenz repliziert werden. ten“ (Mitgestalten) und „Bin interessiert“ (Interesse) (CSSM: Continuous State-Sampling Method)

Erfassung der didaktischen Schwerpunktsetzung mittels Unterrichtsbeobachtung (, Außensicht")

Zur Erfassung der didaktischen Schwerpunktsetzungen dient die Methode der indirekten Beobachtung. Hierbei werden Lehr-Lern-Prozesse zunächst aufgezeichnet und erst anschließend bewertet (Mayring 1996). Hinsichtlich der Kategorie "unterrichtliche Arbeitsform" wurde zwischen Frontalunterricht und schulerzentrierten Arbeitsphasen differenziert. Letztere sind dadurch gekennzeichnet, dass Lehrpersonen nach dem Bereitstellen der Unterrichtsmaterialien nur noch einen vergleichsweise geringen Einfluss auf die Lerntätigkeit nehmen, wohingegen im Frontalunterricht eine lehrende Person (dies kann z. B. im Rahmen von Ergebnispräsentationen auch ein Schüler sein) den Unterrichtsablauf steuert und kontrolliert.

Erfassung der individuellen Konstitution

Bezuglich der Fragestellung zum subjektiven Erleben des Unterrichts in Abhängigkeit von der individuellen Konstitution beschränken wir uns exemplarisch auf eine Unterscheidung zwischen Schulern mit hoher vs. geringer Ausprägung hinsichtlich der Selbstwirksamkeitserwartungen. Dabei geht es um die persönliche Einschätzung der eigenen Kompetenzen, Schwierigkeiten und Barrieren beim Lernen zu bewältigen (Bandura 1982, 1989; Schwarzer 1992). Es liegt auf der Hand, dass insbesondere in Lehr-Lern-Settings, die die Eigenaktivität der Lerner sowie die Verantwortungsubernahme für den eigenen Wissenserwerb betonen, diese Erwartungen eine wichtige personale Ressource darstellen. Bei der Bewältigung von Aufgaben und Problemen wägt man die Anforderungen und die Kompetenzen (unter Einbezug förderlicher oder hinderlicher Faktoren der Lernumwelt) ab und entscheidet dann über die Handlung bzw. Bewältigungsreaktion. Selbstwirksamkeitsuberzeugungen beeinflussen beispielsweise die Auswahl von Handlungen oder das Ausmaß der Anstrengung und somit indirekt den Handlungserfolg. Es ist auch plausibel, dass Lernende mit vergleichsweise gering ausgeprägten Selbstwirksamkeitsüberzeugungen Lehr-Lern-Situationen anders wahrnehmen als Mitschüler mit einer günstigeren Konstitution. ${ }^{6}$

6 Die Selbstwirksamkeitserwartungen wurden mit Hilfe eines 13 Items umfassenden Fragebogens von Strittmatter et al. (1984) erhoben. 


\section{Empirische Befunde}

\subsection{Methodische Organisation des Unterrichts}

Wie ein Blick auf Tabelle 1 verdeutlicht, herrschen in beiden Klassen schülerzentrierte Unterrichtsformen vor. Dabei erreicht Gruppe 2 mit einem Anteil schülerzentrierter Phasen an der gesamten Unterrichtszeit von fast $70 \%$ einen etwas höheren Wert als Gruppe 1 (65\% der Unterrichtszeit). Im Vergleich zu den in der Literatur berichteten Werten für den herkömmlichen Unterricht kann insbesondere der für das Selbstorganisierte Lernen ermittelte Anteil schülerzentrierter Arbeitsphasen als weit überdurchschnittlich gelten. ${ }^{7}$ Dieser Befund lässt sich u.a. als Beleg dafür deuten, dass das realisierte didaktische Design die Möglichkeit bietet, selbstorganisiertes Lernen anzuregen und zu fördern. Selbstorganisiertes Lernen stellt damit - schon im Hinblick auf die den Schülern eingeräumten Handlungs- und Entscheidungsspielräume - ein Kontrastprogramm zum traditionellen Unterrichtsgeschehen dar.

\begin{tabular}{|c|c|c|c|c|}
\hline \multirow[b]{3}{*}{ Unterrrichtliche Arbeitsform } & \multicolumn{4}{|c|}{ Klasse } \\
\hline & \multicolumn{2}{|c|}{$\begin{array}{c}\text { Klasse 1 } \\
(\text { Sole }+ \text { Bil. }) \\
\end{array}$} & \multicolumn{2}{|c|}{$\begin{array}{c}\text { Klasse } 2 \\
(\text { SoLe + WiR })\end{array}$} \\
\hline & $\begin{array}{c}\text { absolut } \\
\text { (in Min.) }\end{array}$ & $\begin{array}{l}\text { relativ } \\
\text { (in \%) }\end{array}$ & $\begin{array}{l}\text { absolut } \\
\text { (in Min.) }\end{array}$ & $\begin{array}{l}\text { relativ } \\
\text { (in \%) }\end{array}$ \\
\hline Frontalunterricht & 811 & 30,6 & 863 & 28,9 \\
\hline Schülerzentrierte Phasen & 1728 & 65,1 & 2074 & 69,5 \\
\hline Sonstiges & 115 & 4,3 & 47 & 1,6 \\
\hline Gesamt & 2654 & 100,0 & 2984 & 100,0 \\
\hline
\end{tabular}

Tabelle 1: Verteilung der Unterrichtszeit auf unterrichtliche Arbeitsformen

7 Wie beispielsweise eine jungst abgeschlossene Befragung von 177 Lehrkraften und uber 1.400 Schulern aus dem kaufmannisch-verwaltenden Bereich zeigt, dominiert in der schulischen Praxis nach wie vor der fragend-entwickelnde Frontalunterricht; handlungsorientierte Lehr-Lern-Arrangements nehmen eine eher erganzende Funktion ein (Patzold, Klusmeyer, Wingels \& Lang 2003).
3.2 Subjektives Erleben von Lehr-Lern-Situationen in Abhängigkeit von der Unterrichtsorganisation

Aus didaktischer Sicht von unmittelbarem lnteresse ist die Bedeutung unterrichtsorganisatorischer Rahmenbedingungen für das Erleben von Lehr-LernSituationen. Hinsichtlich des motivationalen Erlebens kann angenommen werden, dass das Autonomieerleben und Interesse in Abhăngigkeit von der gewählten unterrichtlichen Arbeitsform und den zu bearbeitenden Lerninhalten variieren. Im Zuge der bereits durchgefürten Prozessanalysen Selbstorganisierten Lernens (s. o.) war postuliert und durch die Gegenüberstellung einer traditionell und einer selbstorganisiert lernenden Untersuchungsgruppe belegt worden, dass eine selbstorganisationsoffene Lernumgebung das Erleben von Autonomie und das Interesse fördert. Vor diesem Hintergrund gilt es, mittels Gegenüberstellung von schülerzentrierten Arbeitsphasen und lehrergesteuertem Frontalunterricht zu uberprufen, wie sich die motivationale Aktivierung wăhrend der verschiedenen Unterrichtsphasen darstellt.

Im Vorfeld der Überprüfung wird zunächst pro Untersuchungsteilnehmer fur jedes Erlebensitem ein Kennwert in Abhängigkeit von der didaktischen Schwerpunktsetzung errechnet. Zur Ermittlung möglicher Unterschiede kann dann für jede Kategorie eine zweifaktorielle Varianzanalyse mit einem Messwiederholungsfaktor (unterrichtliche Arbeitsform: Frontalunterricht vs. schulerzentrierte Arbeitsphase) und einem unabhängigen Faktor (Treatment: Klasse 1 vs. Klasse 2) durchgeführt werden. ${ }^{8}$ Angesichts der bestehenden Zusammenhänge zwischen individuellen Voraussetzungen zu Beginn des Schuljahres (hier sind insbesondere die Unterschiede zwischen den Vergleichsgruppen hinsichtlich des Konstruktes "Selbstwirksamkeits-Erwartungen“" $\mathrm{zu}$ berucksichtigen) und dem subjektiven Unterrichtserleben ${ }^{9}$ entschieden wir uns dafur, jeweils eine Kovarianzanalyse mit Messwiederholung $\mathrm{zu}$ rechnen, um den Einfluss unterschiedlicher Startbedingungen zu neutralisieren (siehe hierzu ausführlich Seifried 2004a).$^{10}$ Für eine erste Interpretation der Ergebnisse der Kovarianzanalyse leistet eine Gegenúberstellung der Mittelwerte fur die einzelnen Arbeitsformen gute Dienste (siehe Abbildung 1). Bei der Interpretation der anschließend dargestellten Analysen geht es nun weniger um die absolute Höhe der Werte, von Interesse ist vielmehr die relative Bewertung der Kategorien im Vergleich zueinander und ob sich

Zu dieser Vorgehensweise siehe auch Wild 2000, S. $48 \mathrm{ff}$

9 Signifikanter Zusammenhang zwischen Selbstwirksamkeitserwartungen und Mitgestalten $\mathrm{r}=.40$ ); hoch signifikanter Zusammenhang zwischen Selbstwirksamkeitserwartungen und Interesse $(r=.46)$.

10 Voraussetzung fur die Kovarianzanalyse ist die Parallelitat der Regressionsgeraden. Diese Annahme ist jeweils erfullt. 
Unterschiede in beiden Klassen in gleicher Form feststellen lassen (z. B.: Liegen die Mitgestaltenswerte für schulerzentrierte Arbeitsphasen in beiden Klassen über den Vergleichswerten für Frontalunterricht?)

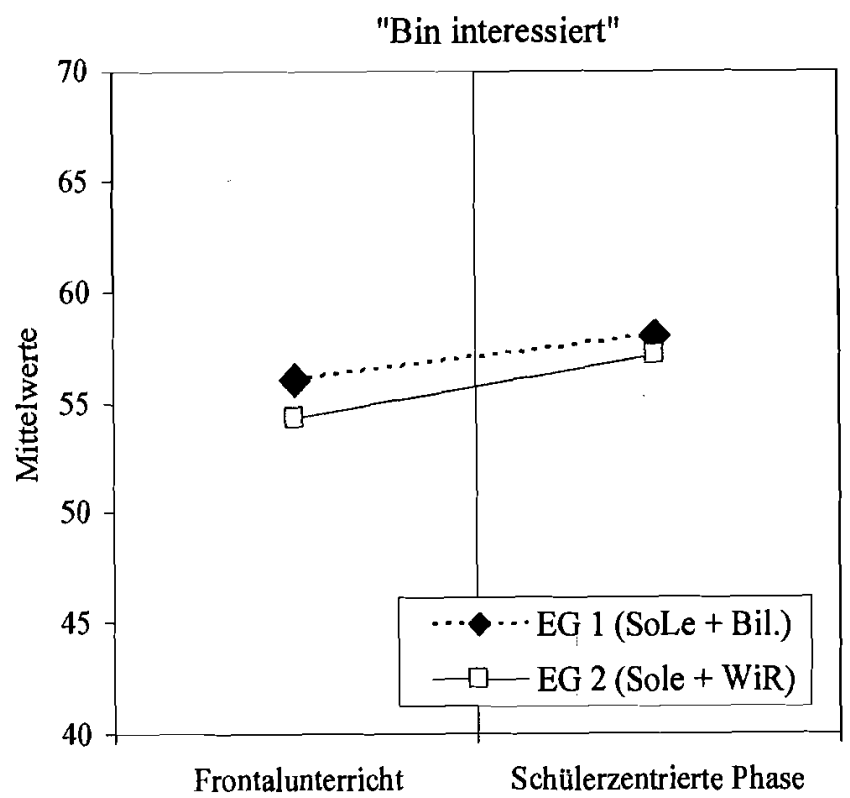

Abbildung 1: Motivationales Erleben des Unterrichts in Abhängigkeit von der unterrichtlichen Arbeitsform (Kovarianzanalyse mit Messwiederholung, Kovariate: Selbstwirksamkeitserwartungen)

Die Analyse der unterrichtlichen Arbeitsformen führt zu den erwarteten Befunden: In beiden Klassen liegt das wahrgenommene Ausmaß an Mitgestaltungsmöglichkeiten während der als schulerzentriert zu charakterisierenden Arbeitsphasen über den Werten für Frontalunterricht. Der Haupteffekt für den Faktor „unterrichtliche Arbeitsform“ ist hoch signifikant und substanziell $\left(F=30.785, p=.000, \eta^{2}=.498\right)$. Im Ausmaß etwas geringer, aber immer noch signifikant und substanziell ist auch der Effekt für das berichtete Interesse $\left(F=8.469, p=.007, \eta^{2}=.215\right)$. Erneut bewegen sich die Mittelwerte in den schülerzentrierten Unterrichtssegmenten über den Vergleichsgrößen für Frontalunterricht.

Zusätzlich wurde überprüft, inwiefern sich die Motivationslagen der beiden hier analysierten SoLe-Klassen im Vergleich zur Lernmotivation einer traditionell lernenden Kontrollgruppe (fragend-entwickelnder Frontalunterricht) darstellen. Zur Beantwortung dieser Fragestellung wurde ein von der Forschergruppe um Prenzel entwickelter Fragebogen eingesetzt, der eine situationsspezifische Erfassung der Lernmotivation sowie der Bedingungen, die die Entwicklung selbstbestimmter Motivation fördern, ermöglicht (Prenzel, Kristen, Dengler, Ettle \& Beer 1996). Es zeigte sich, dass - in Analogie zu den Befunden der in der Vergangenheit durchgefuhrten Prozessanalysen Selbstorganisierten Lernens (s. o.) - beide SoLe-Klassen gegenüber der TraLe-Klasse über Vorteile hinsichtlich „erwünschter" Motivationsarten (intrinsische Motivation und Interesse) verfügen (Seifried 2004a, S. $170 \mathrm{ff}$.). Innerhalb einer selbstorganisationsoffenen Lernumgebung sorgen dann insbesondere die schülerzentrierten Arbeitsphasen für gute motivationale Bedingungen (s. o.).

\subsection{Subjektives Erleben von Lehr-Lern-Situationen in Abhängigkeit von der individuellen Konstitution der Lernenden}

In einem sich anschließenden Analyseschritt geht es nun darum, wie sich die Selbstwirksamkeitserwartungen der Schüler auf das subjektive Erleben der beiden Lehr-Lern-Situationen auswirken. Mittels Mittelwertsplit wurden die Untersuchungsteilnehmer in zwei Gruppen mit niedrigen $(-)$ vs. hohen $(+)$ Selbstwirksamkeitserwartungen eingeteilt. Zur Ermittlung möglicher Effekte dient erneut eine zweifaktorielle Varianzanalyse mit einem Messwiederholungsfaktor (unterrichtliche Arbeitsform: Frontalunterricht vs. schülerzentrierte Arbeitsphase) und einem unabhängigen Faktor (Selbstwirksamkeitserwartungen $=$ SWK: hoch vs. gering).

Fur das Erleben der Lehr-Lern-Situationen aus motivationaler Sicht ergibt sich für „Ich kann mitgestalten" ein Haupteffekt in Abhangigkeit von de unterrichtlichen Arbeitsform ( $F=30.500, p=.000, \eta^{2}=.488$ ). Beide Gruppen berichten über ein höheres $\mathrm{Ma}$ des Mitgestaltens in schulerzentrierten Arbeitsphasen. Ähnlich gelagert ist der Fall für das berichtete Interesse. Erneut ergeben sich signifikante Haupteffekte für die unterrichtliche Arbeitsform $\left(F=8.752, p=.006, \eta^{2}=.215\right)$. Abbildung 2 zeigt die entsprechenden Mittelwerte. 
"Bin interessiert"

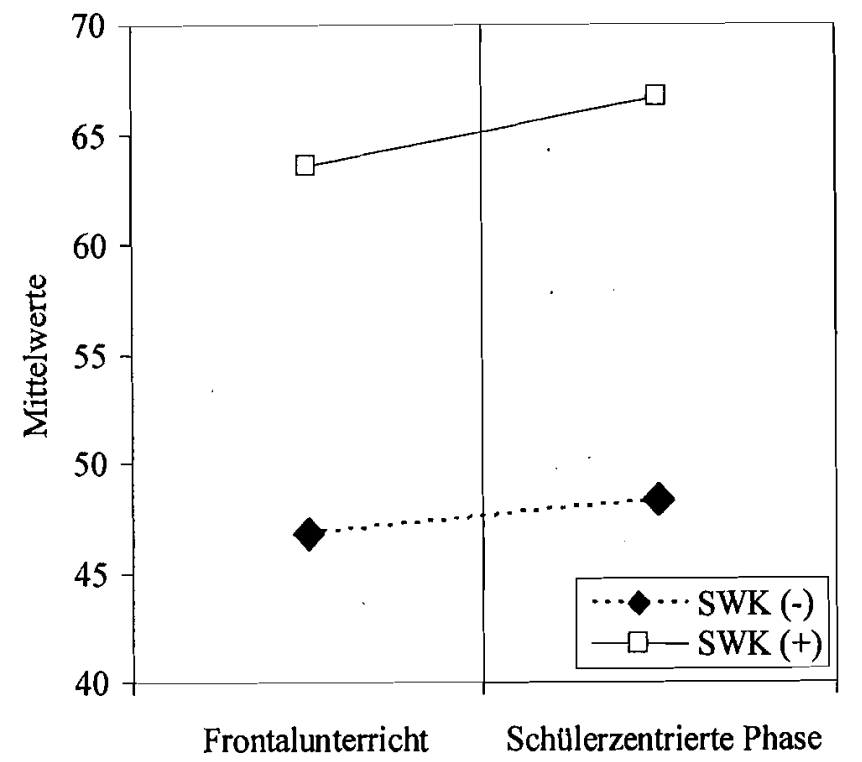

Abbildung 2: Unterrichtserleben in Abhängigkeit von Selbstwirsamkeitserwartungen und der unterrichtlichen Arbeitsform (Varianzanalyse mit Messwiederholung

Für den Faktor "Selbstwirksamkeitserwartungen“ bestehen ebenfalls signifikante Haupteffekte (Mitgestalten: $F=9.661, p=.004, \eta^{2}=.232$; Interesse: $F$ $=10.956, p=.002, \eta^{2}=.255$ ). Für Lerner mit Nachteilen hinsichtlich der individuellen Konstitution stellt sich erwartungsgemäß das Unterrichtserleben deutlich weniger günstig dar als für Lerner, die diesbezüglich uber Vorteile verfügen (siehe Abbildung 2). Die befurchteten Nachteile für schwächere Lerner während offener, durch die Lehrkraft nicht direkt gesteuerte und vorstrukturierte Unterrichtssequenzen lassen sich indes nicht feststellen. Vielmehr ist auch für Schüler mit einer weniger günstigen Konstitution festzuhalten, dass sie diese offene Unterrichtsphasen vergleichsweise positiv erleben.

\section{Diskussion}

Um Zusammenhänge zwischen der beobachtbaren Außensicht und den Schülerselbsteinschătzungen (Innensicht) zu ermitteln, wurde eine empirische Koppelung von Erlebens- und Beobachtungsdaten vorgenommen. Die Analyse des subjektiven Erlebens der Lehr-Lern-Situationen in Abhängigkeit unterrichtlicher Rahmenbedingungen hat gezeigt, dass durch die Unterscheidung verschiedener Arbeitsformen ein beträchtliches Maß der Variabilität der Erlebensdaten im Zeitablauf (Zeitreihen mit ca. 300 Messzeitpunkten) aufgeklärt werden kann. Es soll aber nicht verschwiegen werden, dass man bei der isolierten Betrachtung von unterrichtlichen Arbeitsformen Gefahr läuft, Interdependenżen zu vernachlasssigen und eigentlich bestehende Effekte nicht aufklären zu können.

Insgesamt ergeben sich eine Reihe theoriekonformer Resultate, die die zu Projektbeginn geäußerten Vermutungen weitgehend unterstützen: Es bestehen erwartungsgemăß Wahrnehmungsunterschiede in Abhängigkeit von der unterrichtlichen Arbeitsform (Frontalunterricht vs. schülerzentriertes Arbeiten). Die Schüler beider Klassen berichten in Phasen selbstständiger Schülerarbeit beispielsweise über ein höheres Ausmaß an Mitgestaltungsmöglichkeiten als in lehrerzentrierten Unterrichtsphasen. Dieses Resultat erscheint auf den ersten Blick trivial. Die im Vergleich zum Frontalunterricht höheren Werte bei "Ich kann mitgestalten" im Rahmen schüleraktiver Unterrichtsphasen weisen jedoch darauf hin, dass die Schüler bestehende Freiheitsgrade auch als solche wahrnehmen, und dies im doppelten Wortsinne. Das höhere Ausmaß an Mitgestaltungsmöglichkeiten spiegelt sich theoriekonform auch in den Selbstberichten bezuglich des in der jeweiligen LehrLern-Situation aktuellen Interesses wider: Die Schülervoten während der schülerzentrierten Arbeitsphasen liegen über den Vergleichswerten für den Frontalunterricht. Die berichteten Zusammenhänge sind daher als weiteres Indiz für die motivationsfordernde Wirkung einer aktiven und selbstbestimmten Auseinandersetzung mit Lerninhalten zu werten. Die theoretisch begründeten Annahmen uber systematische Zusammenhänge zwischen motivationsrelevanten Bedingungen und dem motivationalen Erleben des Unterrichts werden durch die vorliegenden Befunde somit unterstutzt.

Bezuglich des subjektiven Erlebens von Lehr-Lern-Situationen in Abhängigkeit von Selbstwirksamkeitserwartungen zeigt sich, dass Lerner mit einer ungünstigeren Konstitution den Unterricht deutlich weniger positiv wahrnehmen als Schüler mit guten Startbedingungen. Dies gilt sowohl für 
lehrer- als auch für schulerzentrierte Arbeitsphasen. Ungeachtet dessen lässt sich feststellen, dass auch Schuler mit geringen Selbstwirksamkeitserwartungen offene Unterrichtssequenzen aus motivationaler Sicht als vorteilhaft wahrnehmen. Die nicht selten propagierten Nachteile offener Unterrichtssequenzen für Schüler mit geringen Selbstwirksamkeitserwartungen haben sich jedoch nicht gezeigt.

Die berichteten Zusammenhänge zwischen individueller Konstitution und Lernmotivation verweisen auf die Bedeutung einer sorgfältigen Planung komplexer Lehr-Lern-Arrangements unter explizitem Einbezug der individuellen Konstitution von Lernenden. Dieser Planungsschritt sollte also fur Lehrpersonen keine vernachlässigbare „Pflichtübung" darstellen, die nach Beendigung des Vorbereitungsdienstes der Vergangenheit angehört. Weiterhin gilt es, die Betreuungsqualität während der Schülerselbsttätigkeit zu verbessern. Das angestrebte dosierte Entlassen der Lernenden in die Selbstständigkeit („fading") bedeutet keinesfalls, Lerner (und insbesondere Lerner mit einer weniger glunstigen individuellen Konstitution) ohne Unterstutzung durch die Lehrperson sich selbst zu uberlassen. Als vorteilhaft erweist sich die Möglichkeit der individuellen Betreuung unter Berücksichtigung der jeweiligen individuellen Konstitution, die sich in dieser Form im Frontalunterricht als nur schwer realisierbar erweist. Lehrpersonen können sich angesichts der Dezentralisierung der Lehr-Lern-Prozesse gezielt um einzelne Schüler kümmern und finden Zeit für die persönliche Beratung. Schülern fällt es im persönlichen Gespräch mit der Lehrkraft i. d. R. leichter als im Plenum, Fragen zu stellen und bei Unklarheiten mehrfach nachzuhaken (Seifried \& Sembill, im Druck). Dezentrale Lehr-Lern-Arrangements sollten somit für alle Schüler eine gute Möglichkeit zur Kompetenzentwicklung darstellen.

\section{Literatur}

Bandura, A. (1982): Self-efficacy mechanisms in human agency, in: American Psychologist, 37, pp. 122-147.

Bandura, A. (1989): Self-regulation of motivation and action through internal standards and goal systems, in: Pervin, L. A. (Ed.): Goal concepts in personality and social psychology, Hillsdale (NJ): Erlbaum, pp. 19-85.

Berliner, D. C. (1983): Developing conceptions of classroom environments: Some light on the T in classroom studies of ATI, in: Educational Psychologist, 18, pp. 1-13.

Csikszentmihalyi, M. \& Larson, R. (1987): Validity and reliability of the experience-sampling method, in: The Journal of Nervous and Mental Disease, Vol. 175, No. 9, pp. 526-536.

Damasio, A. R. (1999): Descartes' Irrtum. Fühlen, Denken und das menschliche Gehirn, 4., unveränd. Aufl., München: Deutscher Taschenbuch Verlag.

Helmke, A. \& Weinert, F. E. (1997). Bedingungsfaktoren schulischer Leistungen, in: Weinert, F. E. (Hrsg.): Enzyklopädie der Psychologie, Band 3 (Psychologie der Schule und des Unterrichts). Göttingen: Hogrefe, S. 71-176.

Mayring, Ph. (1996): Einführung in die qualitative Sozialforschung. Eine Anleitung zu qualitativem Denken, 3., überarb. Aufl., Weinheim \& Bern: Beltz.

Meyer, H. (1994): Unterrichtsmethoden. 1. Theorieband, Frankfurt/Main: Cornelsen Scriptor.

Nickolaus, R. \& Bickmann, J. (2002): Kompetenz- und Motivationsentwicklung durch Unterrichtskonzeptionsformen. Erste Ergebnisse einer empirischen Untersuchung bei Elektroinstallateuren, in: Die Berufsbildende Schule, 54, S. 236-243.

Pătzold, G., Klusmeyer, J., Wingels, J. \& Lang, M. (2003): Lehr-LernMethoden in der beruflichen Bildung. Eine empirische Untersuchung in ausgewăhlten Berufsfeldern: Universităt Oldenburg.

Preiß, P. (1999): Didaktik des wirtschaftsinstrumentellen Rechnungswesens, München \& Wien: Oldenbourg.

Prenzel, M., Kristen, A., Dengler, P., Ettle, R. \& Beer, T. (1996): Selbstbestimmt motiviertes und interessiertes Lernen in der kaufmännischen Erstausbildung, in: Beck, K. \& Heid, H. (Hrsg.): Lehr-Lern-Prozesse in der kaufmännischen Erstausbildung. Wissenserwerb, Motivierungsgeschehen und Handlungskompetenzen. Zeitschrift fur Berufs- und Wirtschaftspädagogik, Beiheft 13, Stuttgart: Franz Steiner, S. 108-127.

Roth, G. (2001): Fühlen, Denken, Handeln. Die neurobiologischen Grundlagen des menschlichen Verhaltens, Frankfurt: Suhrkamp. 
Santjer-Schnabel, I. (2002): Emotionale Befindlichkeit in einer selbstorganisationsoffenen Lernumgebung. Überlegungen für die ergănzende Berücksichtigung physiologischer Aspekte, Hamburg: Dr. Kovač.

Schumacher, L. (2002): Emotionale Befindlichkeit und Motive in Lerngruppen, Hamburg: Dr. Kova.

Schwarzer, R. (1992) (Ed.): Self-efficacy: Thoughtcontrol of action, Washington (DC): Hemisphere.

Seidel, T. (2003): Lehr-Lernskripts im Unterricht: Freiräume und Einschränkungen für kognitive und motivationale Lernprozesse - eine Videostudie im Physikunterricht, Münster, New York, München \& Berlin: Waxmann.

Seifried, J. (2004a): Fachdidaktische Variationen in einer selbstorganisationsoffenen Lernumgebung - Eine empirische Untersuchung im Rechnungswesenunterricht. Wiesbaden: Deutscher Universitäts-Verlag.

Seifried, J. (2004b): Der Einfluss didaktischer Schwerpunktsetzungen auf das motivationale Erleben des Rechnungswesenunterrichts in einer selbstorganisationsoffenen Lernumgebung, in: Zeitschrift für Berufs- und Wirtschaftspädagogik, 100, S. 242-255.

Seifried, J., Brouër, B. \& Sembill, D. (2002). Was lernen Schülerinnen und Schüler im selbstorganisationsoffenen Rechnungswesenunterricht?, in: Zeitschrift für Berufs- und Wirtschaftspädagogik, 98. Bd., H. 4, 574593.

Seifried, J. \& Sembill, D. (im Druck): Schülerfragen - ein brachliegendes didaktisches Feld, in: Zeitschrift für Berufs- und Wirtschaftspädagogik.

Sembill, D. (1996): Systemisches Denken, Selbstorganisiertes Lernen, Ganzheitliches Handeln - Systemtheoretische Reflexionen und erziehungswissenschaftliche Umsetzungen, in: Beck, K., Deißinger, T., Müller, W. \& Zimmermann, M. (Hrsg.): Berufserziehung im Umbruch. Didaktische Herausforderungen und Ansatze zu ihrer Bewältigung, Weinheim: Deutscher Studien Verlag, S. 61-78.

Sembill, D. (2000): Selbstorganisiertes und Lebenslanges Lernen, in: Achtenhagen, F. \& Lempert, W. (Hrsg.): Lebenslanges Lernen - seine Grundlegung im Kindes- und Jugendalter, Band 4: Formen und Inhalte von Lernprozessen, Opladen: Leske + Budrich, S. 60-90.

Sembill, D., Wolf, K. D., Wuttke, E., Santjer, I. \& Schumacher, L. (1998): Prozessanalysen Selbstorganisierten Lernens, in: Beck, K. \& Dubs, R. (Hrsg.): Kompetenzerwerb in der Berufserziehung - Kognitive, motivationale und moralische Dimensionen kaufmännischer Qualifizierungsprozesse. Zeitschrift für Berufs- und Wirtschaftspadagogik, Beiheft Nr. 14. Stuttgart: Steiner Verlag, S. 57-79.

Sembill, D., Schumacher, L., Wolf, K. D., Wuttke, E. \& Santjer-Schnabel, I. (2001): Förderung der Problemlösefähigkeit und der Motivation durch Selbstorganisiertes Lernen, in: Beck, K. \& Krumm, V. (Hrsg.): Lehren und Lernen in der beruflichen Erstausbildung. Grundlagen einer modernen kaufmännischen Berufsqualifizierung. Opladen: Leske + Budrich, $\mathrm{S}$ 257-281.

Strittmatter, P., Bederdorfer, H.-W., Bohse-Wagner, N., Götzmann, W. \& Schmitt, D. (1984): Forschungsbericht aus dem Projekt: Konkretisierung, Durchführung und Evaluation pädagogischer Maßnahmen zum Abbau von Angst in schulischen Leistungssituationen, Saarbrücken.

Wild, K.-P. (2000): Der Einfluss von Unterrichtsmethoden und motivationale Orientierungen auf das kognitive Engagement im Berufsschulunterricht in: Duit, R. \& von Rhöneck, C. (Hrsg.): Ergebnisse fachdidaktischer und psychologischer Lehr-Lern-Forschung. Beiträge zu einem Workshop an der Pädagogischen Hochschule Ludwigsburg, Kiel: IPN, S. 35-54.

Wild, K.-P. \& Krapp, A. (1996): Lernmotivation in der kaufmännischen Erstausbildung, in: Beck, K. \& Heid, H. (Hrsg.): Lehr-Lern-Prozesse in der kaufmannischen Erstausbildung. Wissenserwerb, Motivierungsgeschehen und Handlungskompetenzen. Zeitschrift für Berufs- und Wirtschaftspädagogik, Beiheft 13, Stuttgart: Franz Steiner, S. 90-107.

Wolf, K. D. (2003): Gestaltung und Einsatz einer internetbasierten Lernumgebung zur Unterstutzung selbstorganisierten Lernens, Hamburg: Dr. Kovac.

Wuttke, E. (1999): Motivation und Lernstrategien in einer selbstorganisationsoffenen Lernumgebung. Eine empirische Untersuchung bei Industriekaufleuten. Frankfurt et al.: Lang. 